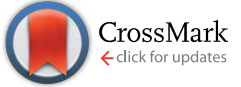

Cite this: RSC Adv., 2017, 7, 3461

Received 16th October 2016 Accepted 7th December 2016

DOI: 10.1039/c6ra25328h

www.rsc.org/advances

\section{Sheathless Dean-flow-coupled elasto-inertial particle focusing and separation in viscoelastic fluid $\dagger$}

\author{
Dan Yuan, ${ }^{a}$ Say Hwa Tan, ${ }^{\mathrm{C}}$ Qianbin Zhao, ${ }^{\mathrm{a}}$ Sheng Yan, ${ }^{\mathrm{a}}$ Ronald Sluyter, ${ }^{\text {de }}$ \\ N. T. Nguyen, ${ }^{C}$ Jun Zhang ${ }^{\star b}$ and Weihua Li ${ }^{\star a}$
}

In this paper, a novel microfluidic device for sheathless particle focusing and separation in viscoelastic fluid is proposed. The device consists of two stages: a straight channel section with asymmetrical expansioncontraction cavity arrays (ECCA section) for sheathless Dean-flow-coupled elasto-inertial particle focusing (1st stage), and a straight channel section for viscoelastic particle separation (2nd stage). In stage 1, particles with diameters of $5 \mu \mathrm{m}$ and $13 \mu \mathrm{m}$ were both focused at the opposite sides of the cavities. Then, the particles were subsequently separated at the 2 nd stage based on the differential focusing dependency on size. The effects of flow rates and channel length on particle separation were investigated. Particle separation in both viscoelastic fluid and Newtonian fluid was also compared to elucidate the differences. In addition, particle separation in the straight channel and integrated ECCA straight channel was also studied. The proposed device was used to separate human Jurkat cells (an immortalized T cell line) and yeast cells. Experimental results show that this technique offers an efficient, continuous, and sheathless particle separation in viscoelastic fluid.

\section{Introduction}

Particle or cell separation in a continuous and label-free manner is essential in a wide range of applications such as disease diagnostics, chemical and biological analysis, and environmental assessment. ${ }^{1-3}$ Centrifugation and filtration are two conventional separation methods. However, the centrifugation method is time-consuming, labour-intensive and may impair the analytes of interest due to the mechanical stress induced by high speed rotation. Filter clogging is an inherent problem which is often difficult to avoid. ${ }^{4}$

In the last two decades, microfluidic devices have been proven as a promising platform for particle/cell manipulation due to their advantages of lower cost, reduced sample volume needed, higher efficiency and accuracy., ${ }^{\mathbf{5} 6}$ According to the operating principle, microfluidic techniques are often classified as either active or passive methods. Active methods are based

${ }^{a}$ School of Mechanical, Materials and Mechatronic Engineering, University of Wollongong, Wollongong, NSW 2522, Australia. E-mail: weihuali@uow.edu.au

${ }^{b}$ School of Mechanical Engineering, Nanjing University of Science and Technology, Nanjing 210094, China. E-mail: junzhang@njust.edu.cn

'Queensland Micro- and Nanotechnology Centre, Griffith University, Brisbane, QLD 4111, Australia

${ }^{d}$ School of Biological Sciences, University of Wollongong, Wollongong, NSW 2522, Australia

'Illawarra Health and Medical Research Institute, Wollongong, NSW 2522, Australia $\dagger$ Electronic supplementary information (ESI) available. See DOI: $10.1039 / \mathrm{c} 6 \mathrm{ra} 25328 \mathrm{~h}$ on the application of external force fields such as acoustic, ${ }^{7}$ dielectrophoretic (DEP), ${ }^{8,9}$ optical $^{10}$ and magnetic ${ }^{11}$ forces. These active methods are limited by high fabrication complexity and expensive auxiliary equipment, although they can provide a more precise manipulation of particles or cells. Passive methods are based on the geometrical effects of microchannels and hydrodynamic forces. Examples of passive methods are pinched flow fractionation, ${ }^{12}$ inertial microfluidics, ${ }^{\mathbf{1 3 - 1 5}}$ deterministic lateral displacement, ${ }^{16}$ microfiltration ${ }^{17}$ and hydrophoresis. ${ }^{\mathbf{8} 18}$

All the passive methods mentioned above have been performed in Newtonian fluid, while particle or cell manipulation in viscoelastic fluid can be more flexible due to the fact that it is much easier to realize three-dimensional focusing in simple straight channels. ${ }^{19}$ Recently, particle manipulation in viscoelastic fluid has gained increasing attention. In a viscoelastic fluid, the first normal stress difference $\left(N_{1}\right)$ induced in the pressure driven flows of dilute polymer solutions can lead to lateral migration of the suspended particles or cells in microfluidic channels. ${ }^{20-22}$ Based on this principle, many studies regarding $3 \mathrm{D}$ particle focusing in viscoelastic fluid have been performed. ${ }^{19,23-30}$ However, few studies have achieved particle separation in viscoelastic fluid. Yang et $a .^{19}$ demonstrated sheathless elasto-inertial particle focusing and continuous separation of different sized particles from the size dependence of the elastic force in a straight rectangular microchannel. Ahn et $a l .{ }^{31}$ achieved "elasto-inertial" particle separation by size in poly(ethylene oxide) (PEO) solution in a square channel. Because of the random initial distribution of particles, their 
approaches are limited by the low separation efficiency. Researchers also found that the effect of elasto-inertia on lateral migration can be further optimized when particles are injected along the channel wall at the entrance. Therefore, sheath flow or specific channel geometry to induce particles to one side of the wall can be applied initially, then separation can be realized downstream. This has been demonstrated in several studies: with the aid of sheath flow to pinch all the particles along one sidewall at the inlet, Nam et al. ${ }^{32}$ realized particle separation with high purity using elasto-inertial effect of PEO solution in a square channel; Lu and Xuan ${ }^{33}$ presented the "elasto-inertial pinched flow fractionation" (eiPFF) method to achieve continuous particle separation in PEO solutions; Kang et al. ${ }^{34}$ achieved particle separation by size in DNA solution; Del Giudice and his coworkers $^{35}$ integrated magnetophoresis with viscoelasticity in polyacrylamide (PAM) solution in a H-shaped channel to achieve separation of magnetic and non-magnetic beads. However, sheath flow is not beneficial for channel parallelization design and high-throughput processing. Instead of using sheath flow, Nam et al. ${ }^{36,37}$ used specific channel geometry to align particles before separation. He used a circular channel followed by a symmetric bifurcation channel and a sudden expansion region to realize the initialization of the particle position and continuous particle separation in elasticity dominant polyvinyl pyrrolidone (PVP) fluid. However, the length of the circular channel section for pre-focusing and straight channel has to be carefully calculated and designed according to particle sizes.

In all the above papers demonstrating particle manipulation in viscoelastic fluid, the blockage ratio $\beta=a / D_{\mathrm{h}}$, where $D_{\mathrm{h}}$ $=2 w h /(w+h)$ is the hydraulic diameter for a rectangular channel with $w$ and $h$ representing the width and height of the channel cross section are below the value of 0.25 . Particle separation is based on the mechanism that the particles are prone to be focused at the centerline of the channel, but the lateral migration speed is highly dependent on particle size because the driving elastic force acting on the particles is proportional to particle sizes, which result in different lateral positions at outlet. However, when the value of blockage ratio $\beta$ is above 0.25 , the effect of normal stresses is strengthened by the effect of the blockage ratio. Unlike the traditional centerline focusing position, the centerline of the channel is no longer a stable equilibrium position, and the particles will be pushed towards the sidewalls. ${ }^{21,38}$ In this way, particles with blockage ratio below $\beta=0.25$ will migrate to centerline of the channel regardless of their initial positions, while particles with blockage ratio above $\beta=0.25$ will be attracted to the sidewalls.

Based on the new separation principle, this paper reports a device consisting of two stages: a straight channel section with asymmetrical expansion-contraction cavity arrays (ECCA section) for sheathless particle initialization (1st stage), and a straight channel section for label free viscoelastic particle separation (2nd stage). Particles with diameter of $5 \mu \mathrm{m}(\beta=$ $0.13)$ and $13 \mu \mathrm{m}(\beta=0.35)$ are separated effectively in this channel. Separation of human Jurkat cells with diameter of 15 $\mu \mathrm{m}(\beta=0.4)$ and yeast cells with diameter of $5 \mu \mathrm{m}(\beta=0.13)$ are achieved as well. To the best of our knowledge, only Liu et al. ${ }^{39}$ realized the size-based separation of particles and cells suspended in viscoelastic fluid in straight microchannels using this new principle. However, the approach was limited as the viscoelastic fluid containing PEO had to be denaturalized through storage at room temperature without exposure in sunlight for 3 months. In the current study, the PEO solution does not need to be denatured. Moreover, with the aid of an expansion-contraction cavity array (ECCA) section, all the particles are focused to a single line along one sidewall due to the Dean-flow-coupled elasto-inertial effects before the separation process. The ECCA pre-focusing section enhanced the separation performance of the straight section, and the newly prepared PEO solution in this channel provided good separation performance.

\section{Theoretical background}

In an ECCA section, three forces affect particles: inertial lift force, elastic force and drag force. 3D particle pre-focusing at opposite cavity side is realized by the synthetic effect of the three forces. In a straight channel section, particles are affected by inertial lift force and elastic force.

\subsection{Inertial lift force}

The shear gradient lift force and wall lift force are the two dominant forces to govern the particle migration in Newtonian fluids, and equilibrium positions are created by the balance of the two lift forces. The sum of the two inertial lift forces, which is called the net inertial lift force, can be expressed as: $:^{\mathbf{4 0 , 4 1}}$

$$
\begin{gathered}
F_{\mathrm{L}}=\frac{\rho_{\mathrm{f}} U_{\mathrm{m}}{ }^{2} a^{4}}{D_{\mathrm{h}}{ }^{2}} f_{\mathrm{L}}\left(R_{\mathrm{c}}, x_{\mathrm{c}}\right) \\
R_{\mathrm{c}}=\frac{\rho_{\mathrm{f}} U_{\mathrm{m}} D_{\mathrm{h}}}{\mu_{\mathrm{f}}}=\frac{2 \rho_{\mathrm{f}} Q}{\mu_{\mathrm{f}}(w+h)}
\end{gathered}
$$

where $\rho_{\mathrm{f}}, U_{\mathrm{m}}$ and $\mu_{\mathrm{f}}$ are the fluid density, mean velocity, and dynamic viscosity, respectively; $a$ is the spherical diameter of the particles; $D_{\mathrm{h}}=2 w h /(w+h)$ is the hydraulic diameter for a rectangular channel with $w$ and $h$ the width and height of the channel cross section. $Q$ is the volumetric flow rate. The lift coefficient of net inertial lift force $f_{\mathrm{L}}\left(R_{\mathrm{c}}, x_{\mathrm{c}}\right)$ is a function of the position of the particles within the cross section of channel $x_{\mathrm{c}}$ and the channel Reynolds number $R_{\mathrm{c}}{ }^{41}$

\subsection{Elastic force}

In non-Newtonian viscoelastic fluid, particles are affected by additional elastic force, which is determined by the intrinsic properties of the medium. The elastic effects of a nonNewtonian fluid in the channel flow can be characterized by $W_{\mathrm{i}}{ }^{19}{ }^{19}$ which is defined as the ratio between two time constants:

$$
W_{\mathrm{i}}=\frac{\lambda}{t_{\mathrm{f}}}=\lambda \dot{\gamma}=\lambda \frac{2 V_{\mathrm{m}}}{w}=\frac{2 \lambda Q}{h w^{2}}
$$

where $\lambda$ is the relaxation time of the fluid, $V_{\mathrm{m}}$ and $t_{\mathrm{f}}$ are the average velocity and characteristic time of the channel flow, respectively. The characteristic time is approximately equal to 
the inverse of the average (characteristic) shear rate $\dot{\gamma}$, which is $2 V_{\mathrm{m}} / w$ or $2 \lambda Q / h w^{2}$ in a rectangular channel. In viscoelastic fluid, both the first and second normal stresses, $N_{1}\left(=\tau_{x x}-\tau_{y y}\right)$ and $N_{2}$ $\left(=\tau_{y y}-\tau_{z z}\right)$ contribute to particle migration. $\tau_{x x}, \tau_{y y}$, and $\tau_{z z}$ are normal stresses that are exerted in the flow, for the velocity gradient and vorticity direction, respectively. Because $N_{1}$ is much larger than $N_{2}$, the effects of $N_{2}$ can be neglected in diluted PEO solutions. ${ }^{42,43}$ The elastic force $F_{\mathrm{E}}$ originates from an imbalance in the distribution of $N_{1}\left(=\tau_{x x}-\tau_{y y}\right)$ over the size of the particle. ${ }^{44}$

$$
F_{\mathrm{E}} \sim a^{3} \nabla N_{1}=a^{3}\left(\nabla \tau_{x x}-\nabla \tau_{y y}\right)
$$

\subsection{Drag force}

A drag force arises when an object moves through a fluid or when the fluid flows past an object, due to a velocity difference between particle and fluid, and it is expressed as: ${ }^{15,41}$

$$
F_{\mathrm{D}}=6 \pi \mu_{\mathrm{f}} a\left(v_{\mathrm{f}}-v_{\mathrm{p}}\right)
$$

where $v_{\mathrm{f}}$ and $v_{\mathrm{p}}$ are the velocities of the fluid element and particles, respectively.

\section{Materials and methods}

\subsection{Design and fabrication of a microfluidic device}

The microfluidic device has one inlet and two outlets, and comprised a 1st ECCA stage for sheathless particle initialization along the opposite cavity side, and a 2nd straight channel stage for viscoelastic particle separation. In the ECCA section, the right angled isosceles triangular cavities are patterned on one side of a straight channel. The longest edge of the triangle is $900 \mu \mathrm{m}$, and the space between two adjacent cavities is uniform at $900 \mu \mathrm{m}$. Two stages have a uniform cross section of $50 \mu \mathrm{m} \times 30 \mu \mathrm{m}$ (width $\times$ height). The channel lengths in the 1st and 2nd stages are $3 \mathrm{~cm}$ and $2 \mathrm{~cm}$ respectively.

The device was fabricated using standard photolithography and soft lithography techniques. ${ }^{45,46}$ This fabrication included rapid prototyping on a silicon master, and polydimethylsiloxane (PDMS) replica molding and sealing through plasma oxidation.

\subsection{Preparation and rheology of the PEO solution}

PEO (2000 kDa; Sigma-Aldrich) was diluted to $1000 \mathrm{ppm}$ in deionized water (DI water) containing $0.01 \%$ (v/v) Tween 20 (Sigma-Aldrich) (PEO solution). Tween 20 was included in the PEO solution to prevent particle aggregation. For Jurkat cell and yeast separation, PEO was added to a phosphate-buffered saline (PBS) (Sigma-Aldrich) at $1000 \mathrm{ppm}$.

The rheological property of the fluid was measured by a rotational rheometer (Antonpaar MCR 301) that has a parallel plate configuration and a diameter of $20 \mathrm{~mm}$. The experiment was performed at room temperature $\left(24 \pm 1{ }^{\circ} \mathrm{C}\right)$. Fig. S1† shows the viscosity of the $1000 \mathrm{ppm}$ PEO solution as a function of the shear rate. According to Fig. S1, $\uparrow$ the estimated viscosity of $1000 \mathrm{ppm}$ PEO solution is $3 \times 10^{-3} \mathrm{~Pa}$ s. The relaxation time was $12.4 \mathrm{~ms}^{47,48} R_{\mathrm{c}}$ and $W_{\mathrm{i}}$ for $1000 \mathrm{ppm}$ PEO solution from flow rate $Q=5 \mu \mathrm{lmin}^{-1}$ to $Q=20 \mu \mathrm{l} \mathrm{min}^{-1}$ were in the ESI Table $1 . \dagger$

\subsection{Particle preparation}

Particle suspension was prepared by diluting $5 \mu \mathrm{m}$ internally green dyed fluorescent polystyrene microspheres (ThermoFisher Scientific, CV 5\%), and $13 \mu \mathrm{m}$ internally red dyed fluorescent polystyrene microspheres (Thermo Fisher Scientific, CV $5 \%$ ) in the $1000 \mathrm{ppm}$ PEO solution. For comparison, the $5 \mu \mathrm{m}$ and $13 \mu \mathrm{m}$ particles are diluted in DI water as well. The blockage ratio for $5 \mu \mathrm{m}$ and $13 \mu \mathrm{m}$ particles is 0.13 and 0.35 , respectively.

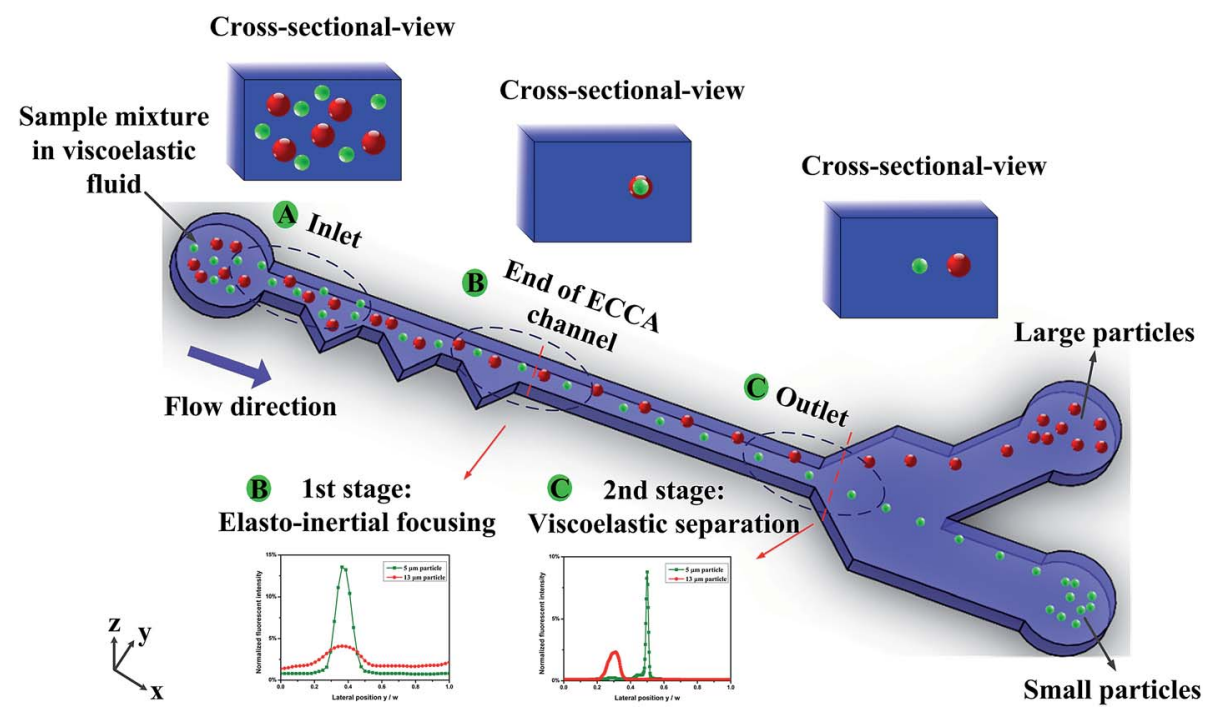

Fig. 1 Schematic of the device for sheathless particle viscoelastic focusing and separation. 
For cell experiments, Jurkat cells (ATCC), an immortalized human $\mathrm{T}$ cell line (average diameter around $15 \mu \mathrm{m}$ ), were cultured in Roswell Park Memorial Institute (RPMI) 1640 medium (ThermoFisher Scientific) containing 10\% fetal bovine serum (Bovogen Biologicals) and $2 \mathrm{mM}$ L-glutamine (ThermoFisher Scientific) in a humidified incubator (Thermo Scientific) at $37{ }^{\circ} \mathrm{C}$ and $95 \%$ air/5\% $\mathrm{CO}_{2}$. Before the cell experiments, Jurkat cells were centrifuged $(300 \times g)$ and re-suspended in PBS containing 1000 ppm PEO. Yeast from Saccharomyces cerevisiae (Sigma-Aldrich) was diluted by PBS containing 1000 ppm PEO. Jurkat and yeast cells were mixed to yield final concentrations of $1.6 \times 10^{6}$ cells per $\mathrm{ml}$ and $6 \times 10^{7}$ cells per $\mathrm{ml}$, respectively.
Before commencing each experiment, the particle mixture solutions were re-suspended by vortex and cell samples were manually stirred to provide uniform suspensions.

\subsection{Experimental setup}

The particle and cell suspensions were transferred to $1 \mathrm{ml}$ syringes and then introduced into the microfluidic chip through silicon tubes by Legato 100 syringe pumps (KD Scientific). The microfluidic chip was then placed onto a CKX41 inverted microscope (Olympus), and images were observed and captured using a CCD camera (Optimos, Q-imaging). Images (a)
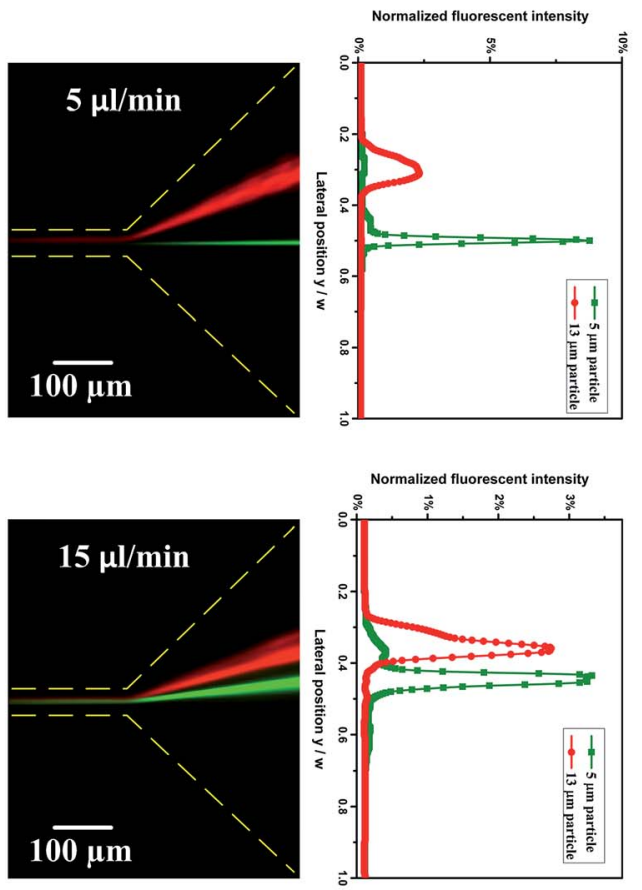
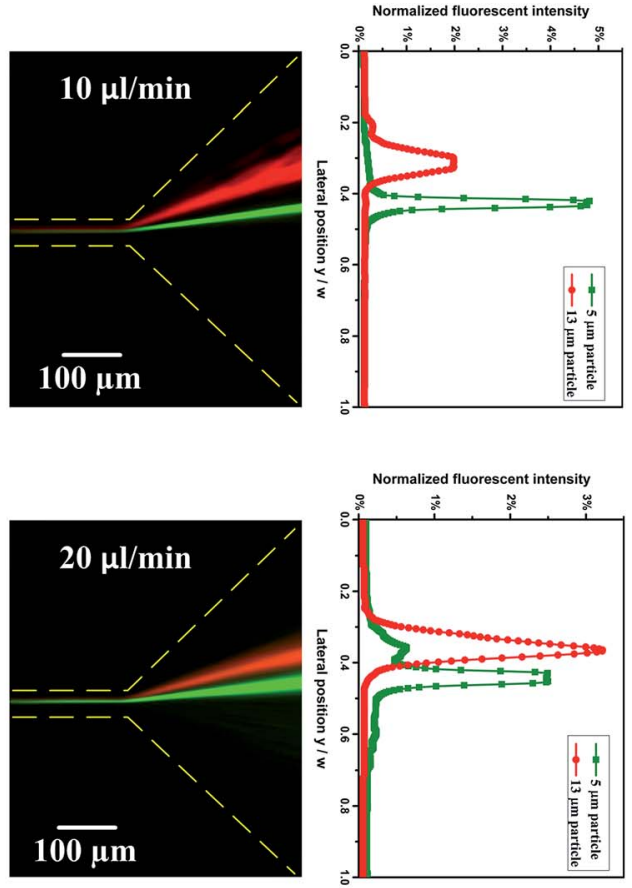

(b)

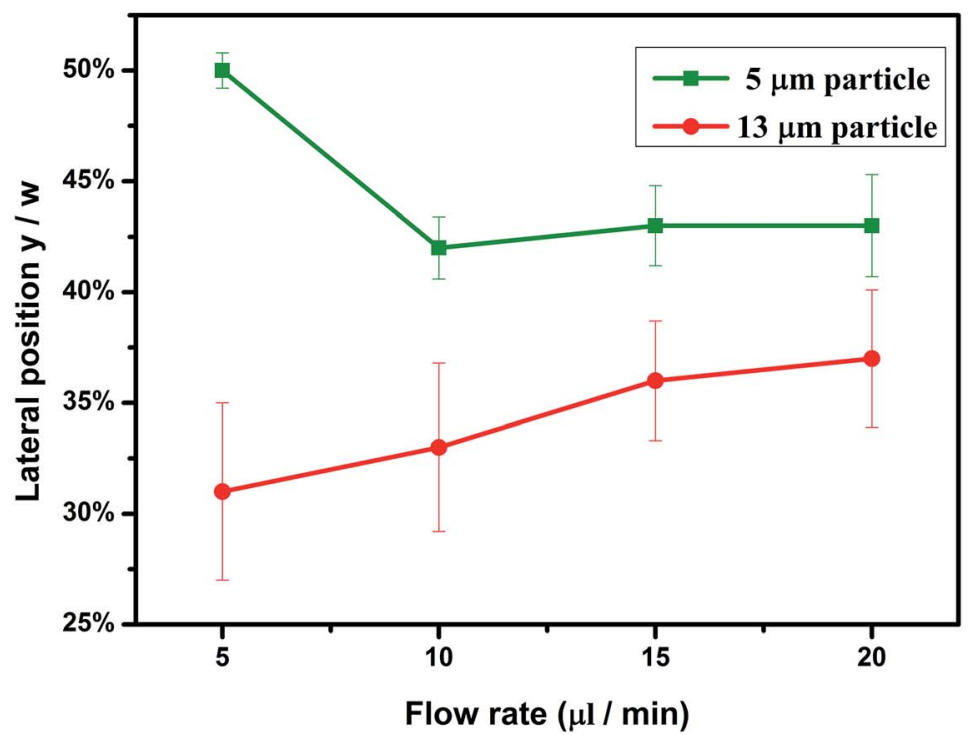

Fig. 2 Distribution of particles at the expansion area at outlet: (a) the captured fluorescent images and corresponding normalized fluorescent intensity profiles at different flow rates; (b) normalized lateral position and width of particles at different flow rates. 
were post-processed and analysed with Q-Capture Pro 7 (Qimaging) software. The cell distributions were analyzed using a customized MATLAB program.

\section{Results and discussion}

\subsection{Schematic of sheathless particle focusing and} separation

Fig. 1 shows a schematic of the device for sheathless particle focusing and viscoelastic separation. The microfluidic device consists of two stages: an ECCA section for sheathless particle initialization (1st stage) and a straight channel section for label free particle separation (2nd stage). At the entrance region of the 1st stage, all particles are randomly distributed. Inertial lift, elastic and Dean drag forces affect the particles flowing through the ECCA section. Therefore, 3D particle pre-focusing at the opposite cavity side is realized by the synthesis effect of the three forces. This can be seen from the top and cross-sectional view at "B end of ECCA channel" section. Then, the particles enter the straight channel section. Since the blockage ratio for larger $13 \mu \mathrm{m}$ particles $(\beta=0.35)$ exceed 0.25 , the particles were displaced from the centerline and the fluid tends to flow through the larger gap between the particles and the wall. Therefore, the enhanced compressive normal stress at the near center side of the particles induced by the intensified shear rates will drive particles towards the sidewalls. ${ }^{21}$ For the smaller $5 \mu \mathrm{m}$ particles $(\beta=0.13)$, the effect of the blockage ratio is small, and particles will migrate to centerline of the channel regardless of their initial positions. This can be seen from the top and the cross-sectional view at "C outlet" section. From the fluorescence intensity profile at the end of first stage (B), the peak of the two curves are overlapped, indicating small and large particles are focused at the same position; at the end of second stage (C), the two curves are split up, indicating the prefocused small and large particles are separated along the lateral direction.

(a)
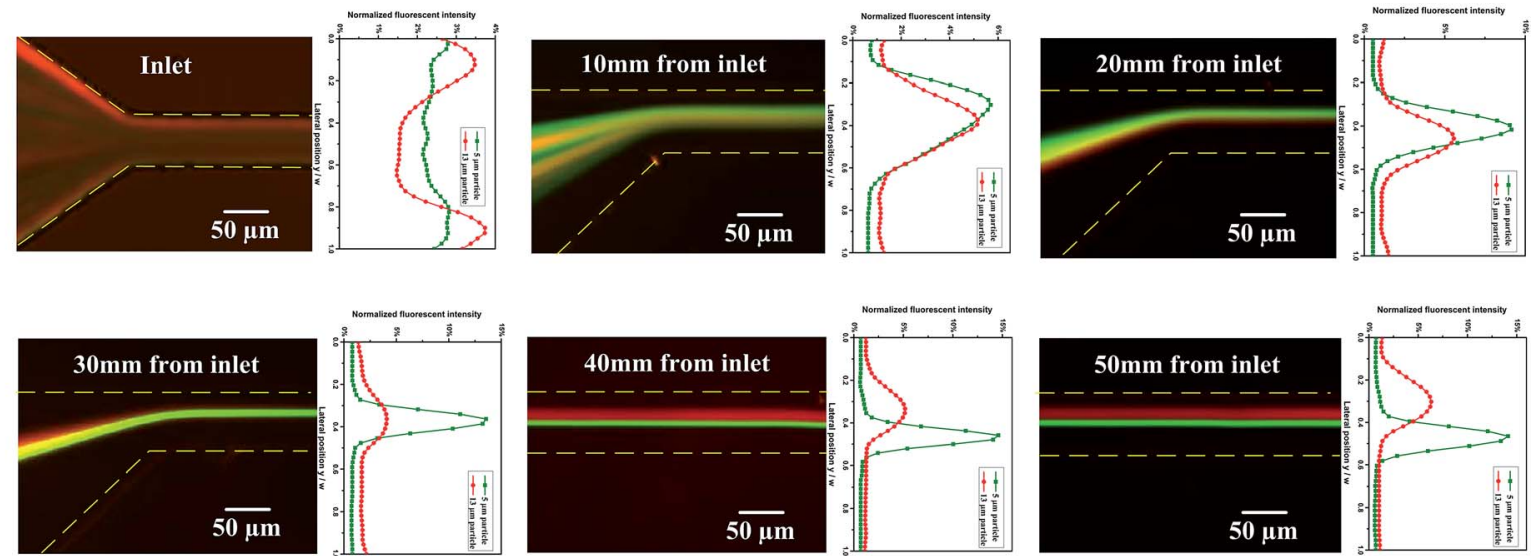

(b)

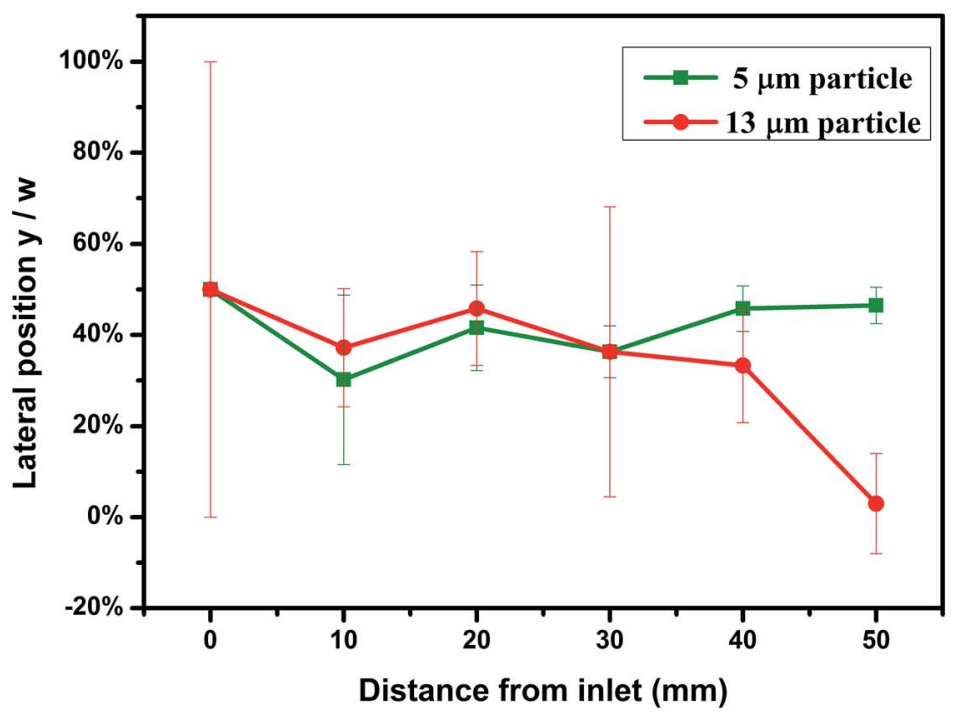

Fig. 3 Distribution of particles at different channel positions: (a) the captured fluorescent images at $Q=5 \mu$ min ${ }^{-1}$ and corresponding normalized fluorescent intensity profiles at different positions; (b) normalized lateral position and focusing width of particles at different distances from inlet. 


\subsection{Effects of flow rates}

The mixture of both $5 \mu \mathrm{m}$ and $13 \mu \mathrm{m}$ particles are focused to a tight stream at the 1st stage; after the initialized particles enter the 2 nd stage, $5 \mu \mathrm{m}$ particles $(\beta=0.13)$ migrate to the center of the channel, while $13 \mu \mathrm{m}$ particles $(\beta=0.35)$ are attracted near the channel walls. As mentioned above, the fluid tends to flow through the larger gap between the particle and the wall when the particle migrates from the centerline, and the enhanced compressive normal stress at the near center side of the particle induced by the intensified shear rates will drive particles towards the side walls. In this channel, the existence of an asymmetrical expansion-contraction cavity arrays in the 1st stage, results in the secondary flow which pushes the particles to the opposite side of cavities. Therefore, only one equilibrium position exists along the channel wall for the $13 \mu \mathrm{m}$ particles in straight channel section at the 2nd stage.

Fig. 2 shows the distribution of particles at the expansion area in the outlet. The captured fluorescent images and corresponding normalized fluorescent intensity profiles at different flow rates are shown in Fig. 2(a). At $Q=5 \mu \mathrm{min}^{-1}\left(R_{\mathrm{c}}=0.7, W_{\mathrm{i}}\right.$ $=28$ ), $13 \mu \mathrm{m}$ particles (red fluorescence line) and $5 \mu \mathrm{m}$ particles (green fluorescence line) are focused well in the Dean-flowcoupled elasto-inertial effect at 1st stage, then they are separated accordingly $(13 \mu \mathrm{m}$ particles are attracted to the upper channel wall, $5 \mu \mathrm{m}$ particles are focused tightly at the centerline). As the flow rate increases to $Q=10 \mu \mathrm{min}^{-1}\left(R_{\mathrm{c}}=1.4, W_{\mathrm{i}}\right.$ $=56)$, there is insufficient time for the $5 \mu \mathrm{m}$ particles to migrate to the centerline of the channel by the elastic force. With the flow rate increasing further $\left(15 \mu \mathrm{lmin}^{-1}\left(R_{\mathrm{c}}=2.1, W_{\mathrm{i}}=84\right)\right.$ and $\left.20 \mu \min ^{-1}\left(R_{\mathrm{c}}=2.8, W_{\mathrm{i}}=112\right)\right)$, the time for particle lateral migration is shorter, therefore the distance between the twosized particles reduces. Moreover, as the flow rate increases, the inertial effect becomes more dominant, thus particles gradually become less focused. As seen from the normalized lateral position and the width of particles stream at different flow rates (Fig. 2(b)), the distance between the normalized lateral positions of two particles becomes smaller as the flow rate increases, and the particles become more dispersed at the same time (the symbols represent the peak fluorescent intensity position of $13 \mu \mathrm{m}$ particles and $5 \mu \mathrm{m}$ particles respectively at each flow rate; the error bars represent the focusing width at $50 \%$ peak fluorescent intensity). The optimal flow rate for particle separation at this integrated ECCA straight channel is $Q$ $=5 \mu \mathrm{l} \mathrm{min}{ }^{-1}\left(R_{\mathrm{c}}=0.7, W_{\mathrm{i}}=28\right)$. The optimal flow rate could be enhanced by extending the straight channel section at the 2 nd stage.

\subsection{Effects of distance from inlet}

Particles have different distributions at varying channel positions, Fig. 3. Fig. 3(a) shows the captured fluorescent images along channel length from inlet at $Q=5 \mu \mathrm{min}^{-1}\left(R_{\mathrm{c}}=0.7, W_{\mathrm{i}}\right.$ $=28$ ) and their corresponding normalized fluorescent intensity profiles. At the inlet, the mixture of $5 \mu \mathrm{m}$ and $13 \mu \mathrm{m}$ particles are randomly distributed. As the particles flow, they are confined within a narrow band at opposite cavity side (10 $\mathrm{mm}$ from inlet). Then, the focusing band becomes narrower at $20 \mathrm{~mm}$ distance (a)

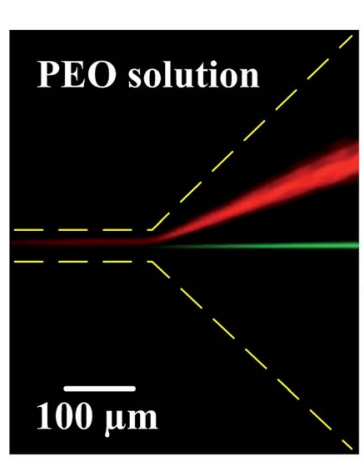

(b)

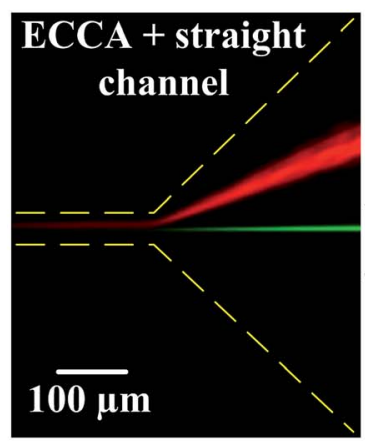

Normalized fluorescent intensity

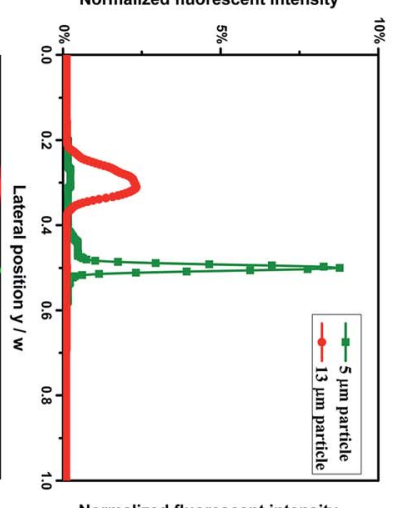

Normalized fluorescent intensity

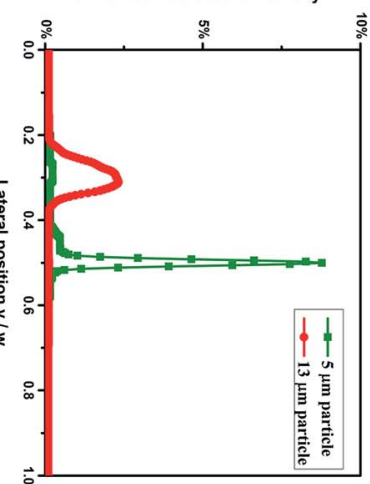

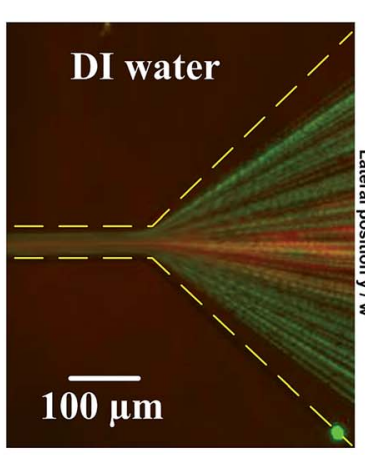

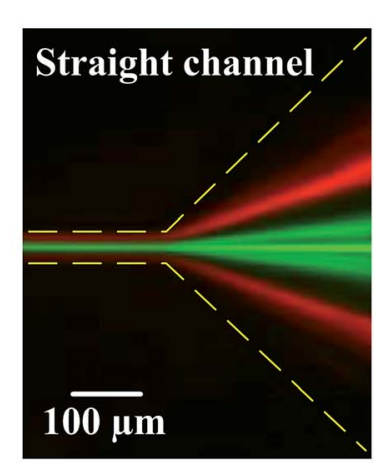

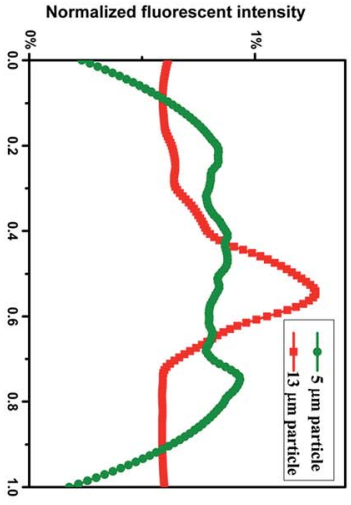

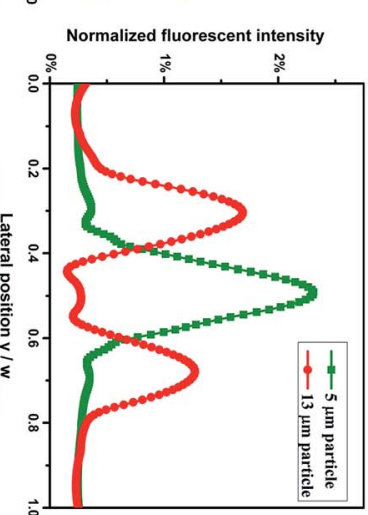

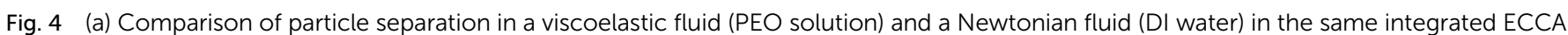

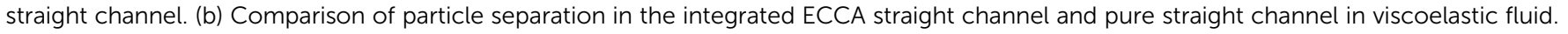


from the inlet. At the end of the 1st stage (30 $\mathrm{mm}$ from inlet), all particles are focused to a tight stream by the Dean-flow-coupled elasto-inertial effects. This can be seen from the overlapping lines in the fluorescent images and the intensity profiles. After the particles enter the straight channel section, $5 \mu \mathrm{m}$ particles migrate to the center of the channel by the elastic force, while 13 $\mu \mathrm{m}$ particles shift to the channel wall ( $40 \mathrm{~mm}$ from inlet) due to the blockage ratio effect. At a position of $50 \mathrm{~mm}$ from the inlet, two different equilibrium positions are formed at outlet and particles with different sizes are separated consequently. It can also be proven from the normalized lateral positions and focusing width of particles at different distances from inlet (Fig. 3(b)), $5 \mu \mathrm{m}$ and $13 \mu \mathrm{m}$ particles came together to a single line at the 1st stage, then migrated to adverse directions and are separated gradually (the symbols represent the peak fluorescent intensity position of $13 \mu \mathrm{m}$ particles and $5 \mu \mathrm{m}$ particles respectively at each position; the error bars represent the focusing width at $50 \%$ peak fluorescent intensity).
4.4. Comparison of particle separation in viscoelastic fluid and Newtonian fluid, in pure straight channel and integrated ECCA straight channel

The particle distribution in a viscoelastic fluid (PEO solution) and a Newtonian fluid (DI water) were compared in the same integrated ECCA straight channel, Fig. 4(a). No particle focusing. is observed at the outlet in a Newtonian fluid. However, singleline focusing is achieved by the combination of inertial force, elastic force and Dean drag force in the viscoelastic fluid. Furthermore, particles with different blockage ratios are successfully separated at the outlet in viscoelastic fluid.

The particle distribution in the integrated ECCA straight channel and the single straight channel in viscoelastic fluid are compared as well, Fig. 4(b). In the pure straight channel, larger particles are focused at the center of narrower channel walls and form two equilibrium positions along the two sides of wider channel walls, but the particles cannot be focused very well. (a)
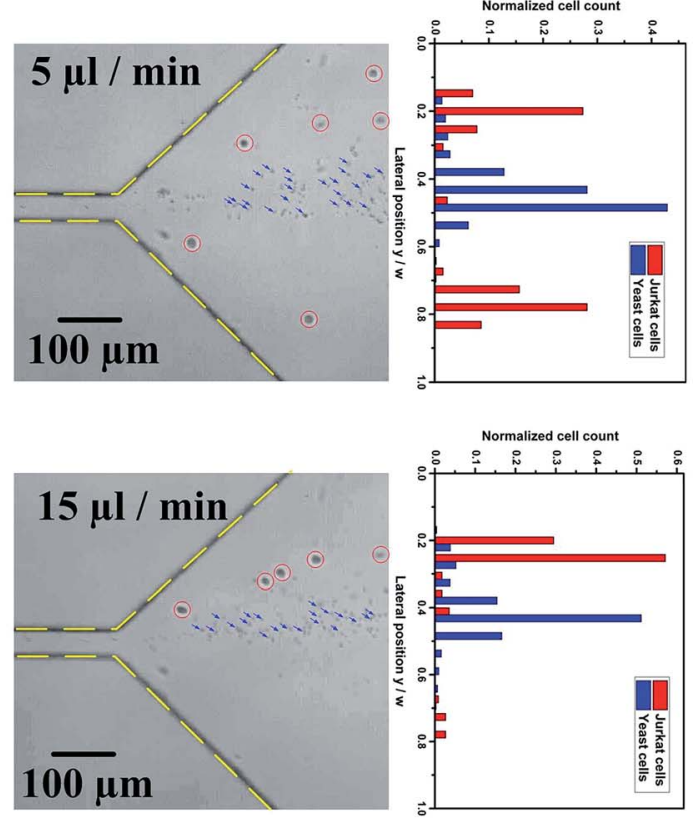
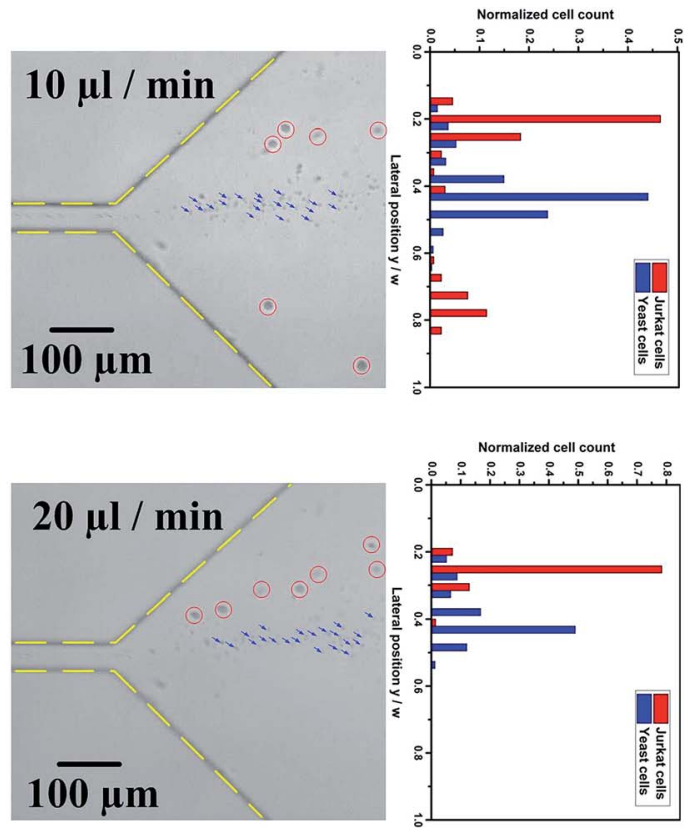

(b)
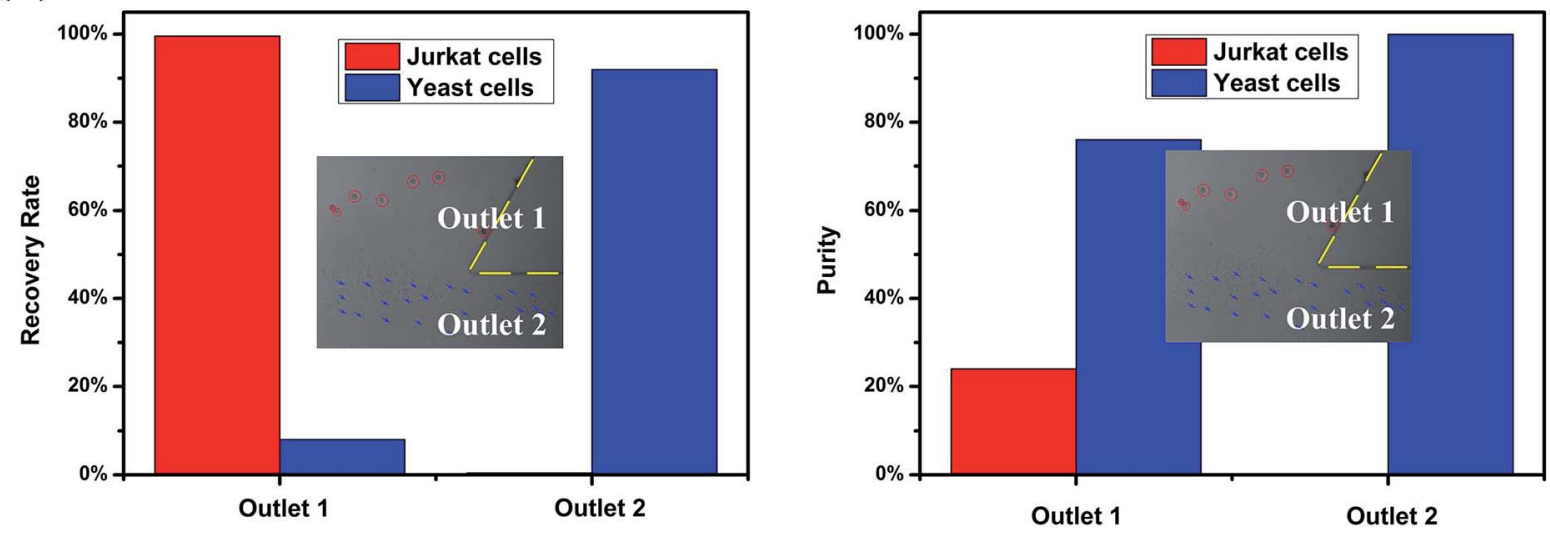

Fig. 5 Separation of Jurkat cells and yeast cells in the viscoelastic fluid. (a) The distribution of Jurkat and yeast cells at the expansion region under different flow rates (captured images in bright field and corresponding normalized cell count). (b) The recovery rate and purity of cells at each outlet at $Q=20 \mu \mathrm{lmin}^{-1}$. 
However, in the integrated ECCA straight channel, the secondary flow reduces two equilibrium positions to one for larger particles. Also, the pre-focusing of particles by the Deanflow-coupled elasto-inertial effect at the 1st stage facilitates particles' distinct lateral migration at the 2nd stage and improves the separation performance significantly.

\subsection{Separation of Jurkat cells and yeast cells}

The previous section demonstrates the separation of $13 \mu \mathrm{m}(\beta=$ $0.35)$ particles and $5 \mu \mathrm{m}(\beta=0.13)$ particles. In order to utilise the potential of our device, separation of Jurkat cells from yeast cells was performed, Fig. 5 . The mean size of Jurkat and yeast cells is about $15 \mu \mathrm{m}(\beta=0.4)$ and $5 \mu \mathrm{m}(\beta=0.13)$, respectively. Fig. 5(a) shows the distribution of Jurkat and yeast cells at expansion region at different flow rates (captured images in bright field and corresponding normalized cell frequency). The red circles and blue arrows indicate the Jurkat cells and yeast cells, respectively. The number of cells for each cell type within different segments in the expansion region was quantified using a customized MATLAB program. The channel width was divided into twenty virtual segments, and the number of cells in each segment was normalized by the total number of cells traversing the entire region. Out of expectation, at $Q=5 \mu \mathrm{l}$ $\min ^{-1}\left(R_{\mathrm{c}}=0.7, W_{\mathrm{i}}=28\right)$, larger cells distributed at both sides of the channel walls, and most smaller cells are in the middle; as the flow rate increases $\left(10 \mu \mathrm{lmin}^{-1}\left(R_{\mathrm{c}}=1.4, W_{\mathrm{i}}=56\right)\right)$, the number of larger cells decreased at the lower channel wall side; with the flow rate increasing to $20 \mu \mathrm{min}^{-1}\left(R_{\mathrm{c}}=2.8, W_{\mathrm{i}}=112\right)$, the separation performance is improved, as well as the focusing performance for both cells. At $Q=20 \mu \mathrm{min}^{-1}\left(R_{\mathrm{c}}=2.8, W_{\mathrm{i}}=\right.$ 112), the Dean-flow-coupled elasto-inertial focusing and separation based on differential focusing for cells with different sizes are achieved in this integrated ECCA straight channel. The optimal separation flow rate for the cells is different from that for particles. This is most likely due to differences in the compressibility of cells and particles, as cell focusing requires a higher flow rate under the Dean-flow-coupled elasto-inertial focusing effect at the 1st stage. Details of the cellular distribution at different channel positions under the flow rate of $20 \mu \mathrm{l}$ $\min ^{-1}$ are summarized in Fig. S2 in ESI. $\dagger$

To evaluate the separation performance of the device, the recovery rate (the ratio of the number of target cells collected in the target outlet to the total number of target cells collected from both outlets), purity (the ratio of the number of target cells to the total number of cells collected at the target outlet), and enrichment ratio (the ratio of the number of target cells to the number of untargeted cells at the inlet divided by the ratio of the number of target cells to the number of untargeted cells collected at the target outlet) were calculated, respectively. The recovery rate, purity and enrichment ratio of Jurkat cells collected at the outlet 1 were $99.6 \%, 24 \%$ and 11.9 , respectively. Fig. 5(b) shows the recovery rate and purity at each outlet at $Q=$ $20 \mu \mathrm{min}^{-1}\left(R_{\mathrm{c}}=2.8, W_{\mathrm{i}}=112\right)$. Since the initial ratio of Jurkat cells in the total cells is only $2.6 \%$, even a small portion of yeast cells mixed with Jurkat cells at the outlet will significantly reduce the purity. However, this device can still have a high recovery rate and enrichment ratio. These results demonstrated that our device can enrich sample concentration significantly and potentially improve the sensitivity of downstream detection.

\section{Conclusion}

In summary, an innovative microfluidic device for sheathless particle focusing and separation in viscoelastic fluid is described in this work. Particles with different blockage ratios are firstly focused on the opposite cavity side by Dean-flowcoupled elasto-inertial effects at the 1st stage, then separated at the 2nd stage based on the differential focusing of particles with different sizes in viscoelastic fluid. The effects of flow rates and distance from the inlet on particle separation were investigated. The comparison of particle separation in a viscoelastic fluid and a Newtonian fluid; in single straight channel and integrated ECCA straight channel were also studied. This device was further applied for separation of Jurkat cells and yeast cells. The recovery rate, purity, and enrichment ratio for Jurkat cells can reach $99.6 \%, 24 \%$ and 11.9 , respectively. The described device offers an efficient, continuous, and sheathless particle separation method in viscoelastic fluid. This device could be potentially used for clinical and biological applications, where biological particles with various sizes need to be separated, or for bio-sample enrichment to improve the sensitivity of the downstream detection unit.

\section{Conflict of interest}

The authors have declared no conflict of interest.

\section{Acknowledgements}

This work is supported by a University of Wollongong - China Scholarship Council joint scholarship. This work was performed in part at the Queensland Node of the Australian National Fabrication Facility, a company established under the National Collaborative Research Infrastructure Strategy to provide nano and micro-fabrication facilities for Australia's researchers. S. H. Tan gratefully acknowledges the support of the Griffith University Post-doctoral fellowship, New Researcher grant, ARC linkage grant (LP150100153) and Griffith UniversityPeking University collaboration grant. The authors are also grateful for Junqi Ma from University of Science and Technology of China doing the cell counting work using Matlab program.

\section{References}

1 N. Pamme, Lab Chip, 2007, 7, 1644-1659.

2 P. Sajeesh and A. K. Sen, Microfluid. Nanofluid., 2014, 17, 152.

3 D. R. Gossett, W. M. Weaver, A. J. Mach, S. C. Hur, H. T. K. Tse, W. Lee, H. Amini and D. Di Carlo, Anal. Bioanal. Chem., 2010, 397, 3249-3267.

4 Z. T. F. Yu, K. M. Aw Yong and J. Fu, Small, 2014, 10, 16871703. 
5 S. Nagrath, L. V. Sequist, S. Maheswaran, D. W. Bell, D. Irimia, L. Ulkus, M. R. Smith, E. L. Kwak, S. Digumarthy and A. Muzikansky, Nature, 2007, 450, 1235-1239.

6 A. A. S. Bhagat, H. Bow, H. W. Hou, S. J. Tan, J. Han and C. T. Lim, Med. Biol. Eng. Comput., 2010, 48, 999-1014.

7 T. Laurell, F. Petersson and A. Nilsson, Chem. Soc. Rev., 2007, 36, 492-506.

8 S. Yan, J. Zhang, G. Alici, H. Du, Y. Zhu and W. Li, Lab Chip, 2014, 14, 2993-3003.

9 Y. Kang, D. Li, S. A. Kalams and J. E. Eid, Biomed. Microdevices, 2008, 10, 243-249.

10 J. Glückstad, Nat. Mater., 2004, 3, 9-10.

11 N. Pamme and C. Wilhelm, Lab Chip, 2006, 6, 974-980.

12 M. Yamada, M. Nakashima and M. Seki, Anal. Chem., 2004, 76, 5465-5471.

13 D. Di Carlo, D. Irimia, R. G. Tompkins and M. Toner, Proc. Natl. Acad. Sci. U. S. A., 2007, 104, 18892-18897.

14 J. Zhang, S. Yan, R. Sluyter, W. Li, G. Alici and N.-T. Nguyen, Sci. Rep., 2014, 4, 4527.

15 J. Zhang, S. Yan, D. Yuan, G. Alici, N.-T. Nguyen, M. E. Warkiani and W. Li, Lab Chip, 2016, 16, 10-34.

16 L. R. Huang, E. C. Cox, R. H. Austin and J. C. Sturm, Science., 2004, 304, 987-990.

17 J. Moorthy and D. J. Beebe, Lab Chip, 2003, 3, 62-66.

18 S. Choi and J.-K. Park, Lab Chip, 2007, 7, 890-897.

19 S. Yang, J. Y. Kim, S. J. Lee, S. S. Lee and J. M. Kim, Lab Chip, 2011, 11, 266-273.

20 G. d'Avino, P. Maffettone, F. Greco and M. Hulsen, J. NonNewtonian Fluid Mech., 2010, 165, 466-474.

21 P. Huang, J. Feng, H. H. Hu and D. D. Joseph, J. Fluid Mech., 1997, 343, 73-94.

22 A. Leshansky, A. Bransky, N. Korin and U. Dinnar, Phys. Rev. Lett., 2007, 98, 234501.

23 F. Del Giudice, G. Romeo, G. D'Avino, F. Greco, P. A. Netti and P. L. Maffettone, Lab Chip, 2013, 13, 4263-4271.

24 E. J. Lim, T. J. Ober, J. F. Edd, S. P. Desai, D. Neal, K. W. Bong, P. S. Doyle, G. H. McKinley and M. Toner, Nat. Commun., 2014, 5, 4120.

25 K. Kang, S. S. Lee, K. Hyun, S. J. Lee and J. M. Kim, Nat. Commun., 2013, 4, 2567.

26 S. Cha, K. Kang, J. B. You, S. G. Im, Y. Kim and J. M. Kim, Rheol. Acta, 2014, 53, 927-933.
27 J. Y. Kim, S. W. Ahn, S. S. Lee and J. M. Kim, Lab Chip, 2012, 12, 2807-2814.

28 D. Yuan, J. Zhang, S. Yan, C. Pan, G. Alici, N.-T. Nguyen and W. Li, Biomicrofluidics, 2015, 9, 044108.

29 G. D'Avino, G. Romeo, M. M. Villone, F. Greco, P. A. Netti and P. L. Maffettone, Lab Chip, 2012, 12, 1638-1645.

30 M. Villone, G. D'Avino, M. Hulsen, F. Greco and P. Maffettone, J. Non-Newtonian Fluid Mech., 2013, 195, 1-8.

31 S. W. Ahn, S. S. Lee, S. J. Lee and J. M. Kim, Chem. Eng. Sci., 2015, 126, 237-243.

32 J. Nam, H. Lim, D. Kim, H. Jung and S. Shin, Lab Chip, 2012, 12, 1347-1354.

33 X. Lu and X. Xuan, Anal. Chem., 2015, 6389-6396.

34 K. Kang, S. S. Lee, K. Hyun, S. J. Lee and J. M. Kim, Nat. Commun., 2013, 4, 2567.

35 F. Del Giudice, H. Madadi, M. M. Villone, G. D'Avino, A. M. Cusano, R. Vecchione, M. Ventre, P. L. Maffettone and P. A. Netti, Lab Chip, 2015, 15, 1912-1922.

36 J. Nam, B. Namgung, C. T. Lim, J.-E. Bae, H. L. Leo, K. S. Cho and S. Kim, J. Chromatogr. A, 2015, 1406, 244-250.

37 J. Nam, Y. Shin, J. K. S. Tan, Y. B. Lim, C. T. Lim and S. Kim, Lab Chip, 2016, 16, 2086-2092.

38 J. Feng, P. Huang and D. Joseph, J. Non-Newtonian Fluid Mech., 1996, 63, 63-88.

39 C. Liu, C. Xue, X. Chen, L. Shan, Y. Tian and G. Hu, Anal. Chem., 2015, 87, 6041-6048.

40 E. S. Asmolov, J. Fluid Mech., 1999, 381, 63-87.

41 D. Di Carlo, Lab Chip, 2009, 9, 3038-3046.

42 J. Magda, J. Lou, S. Baek and K. DeVries, Polymer, 1991, 32, 2000-2009.

43 J. A. Pathak, D. Ross and K. B. Migler, Phys. Fluids, 2004, 16, 4028-4034.

44 K. W. Seo, H. J. Byeon, H. K. Huh and S. J. Lee, RSC Adv., 2014, 4, 3512-3520.

45 E. Sollier, C. Murray, P. Maoddi and D. Di Carlo, Lab Chip, 2011, 11, 3752-3765.

46 J. C. McDonald and G. M. Whitesides, Acc. Chem. Res., 2002, 35, 491-499.

47 X. Lu and X. Xuan, Anal. Chem., 2015, 87, 6389-6396.

48 V. Tirtaatmadja, G. H. McKinley and J. J. Cooper-White, Phys. Fluids, 2006, 18, 043101. 